\title{
Overuse and underuse of pulmonary CT angiography in patients with suspected pulmonary embolism
}

\author{
Hanieh Raji ${ }^{1}$, Seyed Ali JavadMoosavi ${ }^{2}$, Maryam Dastoorpoor ${ }^{1}$, Zahra Mohamadipour ${ }^{3}$, \\ Seyedeh Parvin Mousavi Ghanavati*3
}

\section{Abstract}

Background: The aim of the present study was to evaluate the utilization and diagnostic yields of CT pulmonary angiography (CTPA)using the Revised Geneva score and Wells' criteria, in patients with suspected pulmonary embolism (PE).

Methods: One hundred and twelve adult patients underwent CTPA for suspected PE were participated in this study. The outcome was positive or negative CTPA for PE. Revised Geneva and Wells' scores were calculated. The relationship between the results obtained from these two scores and the available risk factors were compared.

Descriptive analysis such as frequency and mean as well as analytical statistics including chi-square were done. The data analysis was performed using SPSS (v. 22).

Results: In this study, according to the Wells' criteria calculated for the patients, $33.9 \%$ of the patients had low clinical, $56.3 \%$ intermediate and $9.8 \%$ high clinical probability. Among the 11 high clinical patients, $9(81.8 \%)$ were CTPA positive. Based on the revised Geneva score, 65 patients (58\%) had low clinical, $36(32.1 \%)$ intermediate and 11(9.8\%) high clinical probability. Among the 11 high clinical patients, 8 were CTPA positive. Positive predictive value of the low clinical patients based on Wells' criteria and the revised Geneva score was $18.4 \%$ and $30.8 \%$, respectively. Also, positive predictive value for high clinical probability of Wells' criteria and the revised Geneva score was $81.8 \%$ and $72.8 \%$ respectively.

Conclusion: Under/overuse of CTPA in diagnosing PTE is a common problem especially in university hospitals. It is possible to avoid unnecessary CTPA requests using scholarly investigations and more accurate clinical risk assessments.

Keywords: Pulmonary Embolism, Pulmonary CT angiography, Wells’ score, Revised Geneva score

Copyright $@$ Iran University of Medical Sciences

Cite this article as: Raji H, JavadMoosavi SA, Dastoorpoor M, Mohamadipour Z, Mousavi Ghanavati SP. Overuse and underuse of pulmonary CT angiography in patients with suspected pulmonary embolism. Med J Islam Repub Iran. 2018(04 Feb);32:3. https://doi.org/10.14196/mjiri.32.3

\section{Introduction}

Pulmonary thromboembolism (PTE) is a fairly common disease with nonspecific symptoms with potential high risk of mortality and morbidity (1). Diagnosis of PTE includes clinical and laboratory tests based on the available algorithms and criteria such as Wells' criteria and revised Geneva score classifying patients to high, intermediate

Corresponding author: Dr Parvin Mousavi Ghanavati, sp.mg90@yahoo.com

1. Air pollution and Respiratory Diseases Research Center, Ahvaz Jundishapur University of Medical Sciences, Ahvaz, Iran.

2. Faculty of Medicine, Iran University of Medical Sciences and Health Services, Tehran, Iran.

3. Student Research Committee, Ahvaz Jundishapur University of Medical Sciences, Ahvaz, Iran and low clinical probability. CT pulmonary angiography (CTPA) is the most sensitive and precise method in high probability cases $(2,3)$. Because of the non-specific symptoms of the disease, physicians are subject to diagnostic challenges, and there is also the possibility of CTPA request even in low clinical probability cases. According to

$\uparrow$ What is "already known" in this topic:

Diagnosis of PTE includes clinical and laboratory tests based on the available algorithms and criteria such as Wells' criteria and revised Geneva score. CT angiography is overused in the patients with low and intermediate clinical probability.

$\rightarrow$ What this article adds:

In patients with low and intermediate clinical probability, other criteria such as PERC can be considered. If PERC is zero, no $\mathrm{f}$ urther action is needed. If PERC criteria are above zero, a D-Di mer is required. In case of positive D-Dimer, CT angiography is required to be done. 
the related literature, the clinical competence of physicians plays a key role in diagnosing patients properly and selecting diagnostic modalities. Therefore, in university hospitals, where the first clinical study is generally done by novice doctors, there is a possibility of improper decision making with regard to using CTPA, i.e. doing the procedure in unnecessary cases or, otherwise, failure to do it when it is actually required (2-4). In this regard, Crichlow et.al. studied 152 patients with suspected PTE in the Hospital of the University of Pennsylvania, and concluded that $9.2 \%$ and $13.8 \%$ of the CTPA procedures could have been avoided by proper use of PERC and Wells/D-dimer, respectively (5). Molaee et al. in a crosssectional study carried out on 82 patients undergoing CTPA in a university hospital at Tehran University, Iran, reported that in patients with a likely clinical risk the prevalence of PTE was $62.2 \%$. Of those subjects for whom CTPA was requested, the PTE of $54.8 \%$ was unlikely according to modified Wells' criteria. Adherence rate to PTE diagnostic algorithm was only $43.9 \%$. The researchers concluded that there were concerns regarding the excessive request for PTE work up by lessexperienced physicians (6). Choosing CTPA in unnecessary cases imposes considerable financial burdens on both the patients and the health providing center (directly or indirectly through increased hospitalization in the emergency department). Moreover, it exposes the patients to iatrogenic effects, such as increasing risk of malignancy, contrast nephropathy, anaphylactic reactions and effects resulting from false positive cases. By the same token, not using CTPA in necessary cases increases mortality and morbidity risks; thus, further studies on the proper use of this valuable diagnostic method in university hospitals seems necessary $(1,2,5,6)$. To this aim, the present research was a cross-sectional study on patients undergoing CTPA. Based on Wells' criteria and the revised Geneva score, the patients were categorized into three groups of high, intermediate, and low probability. The relationship between the results obtained from these two tests and other risk factors (such as smoking, pregnancy, autoimmune diseases, HTN, OCP use, HRT, obesity, surgery history less than three months, stroke, and central vein catheterization) was studied to examine whether CTPA, as a diagnosis method, had been used properly.

\section{Methods}

This is a cross-sectional analytical descriptive study. The data were collected from medical records of 112 patients underwent pulmonary CT-Angiography as suspected PTE cases at Imam Khomeini Hospital in Ahvaz, southwest of Iran, from February, 2014 to March, 2015. This study was approved by the Ethics Committee of Ahvaz Jundishapur University of Medical Sciences.

CT-angiography interpretation was done by a pulmonologist and a radiologist. The information was collected using a checklist prepared based on Wells' and revised Geneva score. Wells' criteria and the revised Geneva score were calculated for the patients without being aware of their CTPA results. According to Wells' criteria, the patients were classified into less than 2 (low), 2-6 (in- termediate) and above 6 (high), while based on the revised Geneva score, the patients were classified as 0-3 (low), 410 (intermediate) and above 11 (high). Finally, the results obtained from Wells' criteria and the revised Geneva score were compared with the risk factors available in the results of CT-ANGIO (positive vs. negative). The risk factors included: DVT symptoms, possible alternative diagnosis other than pulmonary embolism, immobility, heart rate above 100 beats per minute, history of surgery, DVT, PTE, cancer or hemoptysis. The study also examined PERC (Primary Emboli Rule out Criteria) which included the following variables: age $>50$ years, $\mathrm{HR}>100 / \mathrm{min}, \mathrm{O} 2$ sat on room $>95 \%$, prior history of venous thromboembolism, trauma or surgery within 4 weeks, OCP, HRT and unilateral leg swelling.

\section{Pulmonary CT-angiography}

The CTPA of each patient was done by a fast 16detector computed tomography scanner with 4 detector arrays (Siemens Medical System). The CTPA was performed while the patient lying in the supine position for less than a second. During the imaging procedure, the patients were asked to hold a single breath. Scan volume consisted the entire chest from the lung apex to its base in the cranio-caudal direction. The detector scan area was $40 \mathrm{~mm}$. The slice thickness was $0.625 \mathrm{~mm}$, and the tube current and voltage were $145 \mathrm{ma}$ and $120 \mathrm{kv}$, respectively. Low osmolar nonionic contrast material (135-145ml) was injected through an arm vein during the procedure at a flow rate of $5 \mathrm{ml} / \mathrm{s}$.

\section{Statistical analysis}

To analyze the obtained data, descriptive statistics such as frequency, mean and standard deviation (SD), as well as analytical statistics including chi-square were calculated. A p-value less than 0.05 was considered statistically significant. The data analysis was performed using SPSS (V. 22).

\section{Results}

In this study, the mean age of the participants was $44.1 \pm 4$ (14-91 years), and $76(67.9 \%)$ participants were female of whom 31 (40.8\%) were pregnant. Overally, 44 (39\%) patients were diagnosed as pulmonary embolism. Active DVT was positive for $20(17.9 \%)$ patients with PTE, and past history of DVT was positive in 15 (13.4\%) patients. In addition, no alternative diagnosis was likely other than pulmonary embolism in $59(52.7 \%)$ patients. Five (4.5\%) patients had malignancy, 67 (59.8\%) immobility, and $11(9.8 \%)$ had hemoptysis. More details about the patients are shown in Table 1.

According to Wells' criteria calculated for the patients, $38(33.9 \%)$ patients had low clinical probability, 63 $(56.3 \%)$ with intermediate and 11 patients $(9.8 \%)$ high clinical probability. Among the 11 high clinical patients, 9 (81.8\%) were CTPA positive (Table 2). Moreover, based on the revised Geneva score, $65(58 \%)$ patients were in the low clinical category, $36(32.1 \%)$ in the intermediate and $11(9.8 \%)$ were in the high clinical category. Among the 11 high clinical patients, 8 were CTPA positive (Table 
Table 1. Frequency distribution of clinical variables of the patients based on CT Angiography Results

\begin{tabular}{|c|c|c|c|}
\hline Characteristic & $\begin{array}{c}\text { Total } \\
\mathrm{N}\end{array}$ & $\begin{array}{c}\text { Ct Positive } \\
\text { N (\%) }\end{array}$ & $\begin{array}{c}\text { Ct Negative } \\
\mathrm{N}(\%)\end{array}$ \\
\hline Age $>50$ & $36 / 112$ & $19(52.8 \%)$ & $17(47.2 \%)$ \\
\hline Trauma & $13 / 112$ & $8(61.5 \%)$ & $5(38.5 \%)$ \\
\hline Hypertension & $18 / 112$ & $11(61.1 \%)$ & $7(38.9 \%)$ \\
\hline $\mathrm{OCP}^{*}$ or $\mathrm{HRT}^{* *}$ & $8 / 112$ & $3(37.5 \%)$ & $5(62.5 \%)$ \\
\hline Pregnancy & $31 / 112$ & $4(12.9 \%)$ & $27(87.1 \%)$ \\
\hline Smoking & $20 / 112$ & $10(50.0 \%)$ & $10(50.0 \%)$ \\
\hline Operation in 3 month ago & $34 / 112$ & $14(41.2 \%)$ & $20(58.8 \%)$ \\
\hline Stroke & $4 / 112$ & $4(100.0 \%)$ & $0(0.0 \%)$ \\
\hline Autoimmune disease & $6 / 112$ & $3(50.0 \%)$ & $3(50.0 \%)$ \\
\hline Catheter & 0 & $0(0.0 \%)$ & $0(0.0 \%)$ \\
\hline Deep vein thrombosis & $20 / 112$ & $12(60.0 \%)$ & $8(40.0 \%)$ \\
\hline No other possible diagnosis & $53 / 112$ & $12(22.6 \%)$ & $41(77.4 \%)$ \\
\hline $\mathrm{HR}>100$ & $35 / 112$ & $17(48.6 \%)$ & $18(51.4 \%)$ \\
\hline O2sat $>95 \%$ & $78 / 112$ & $21(26.0 \%)$ & $57(74.0 \%)$ \\
\hline Immobility & $67 / 112$ & $20(29.8 \%)$ & $47(70.2 \%)$ \\
\hline Past history of DVT or PTE & $15 / 112$ & $8(53.3 \%)$ & $7(46.7 \%)$ \\
\hline Cancer & $5 / 112$ & $2(40.0 \%)$ & $3(60.0 \%)$ \\
\hline Hemoptysis & $11 / 112$ & $7(63.6 \%)$ & $4(36.4 \%)$ \\
\hline Leg edema & $20 / 112$ & $12(60.0 \%)$ & $8(40.0 \%)$ \\
\hline Leg pain & $19 / 112$ & $11(57.9 \%)$ & $8(42.1 \%)$ \\
\hline
\end{tabular}

* Oral contraceptive pill

** Hormone replace therapy

Table 2. The relationship between Wells' Criteria and the Revised Geneva Score, and the probability of PTE

\begin{tabular}{|c|c|c|c|}
\hline Wells' Criteria & $\begin{array}{l}\text { CT Positive } \\
(\mathrm{N})\end{array}$ & $\begin{array}{c}\text { CT Negative } \\
(\mathrm{N})\end{array}$ & $\begin{array}{l}\text { Total } \\
(\mathrm{N})\end{array}$ \\
\hline Low & 7 & 31 & 38 \\
\hline Moderate & 28 & 35 & 63 \\
\hline High & 9 & 2 & 11 \\
\hline Total & 44 & 68 & 112 \\
\hline \multicolumn{4}{|c|}{ Revised Geneva Score } \\
\hline Low & 20 & 45 & 65 \\
\hline Moderate & 16 & 20 & 36 \\
\hline High & 8 & 3 & 11 \\
\hline Total & 44 & 68 & 112 \\
\hline
\end{tabular}

2).

Positive and negative predictive values were calculated based on Wells' criteria and the revised Geneva score. As Table 3 shows, positive predictive values of the patients with low clinical probability based on Wells' criteria and revised Geneva score are $18.4 \%$ and $30.8 \%$, respectively. In other words, if the result of Wells' criteria for a patient is "low clinical", the patient is $18.4 \%$ likely to be CTPA- positive.

Pulmonary embolism was also examined in pregnant women. The results obtained from Wells' criteria revealed that 13 patients were in the low clinical category, 18 in moderate, and none was in the high clinical category. Of the pregnant women categorized as moderate clinical, only 4 were CTPA-positive (Table 4). The results of the revised Geneva score are shown in Table 4.

\begin{tabular}{|c|c|c|c|c|c|c|c|}
\hline Wells' Criteria & $\begin{array}{c}\text { CT Positive } \\
(\mathrm{N})\end{array}$ & $\begin{array}{c}\text { CT Negative } \\
(\mathrm{N})\end{array}$ & $\begin{array}{l}\text { Total } \\
(\mathrm{N})\end{array}$ & RevisedGeneva & $\begin{array}{c}\text { CT Positive } \\
(\mathrm{N})\end{array}$ & $\begin{array}{c}\text { CT Negative } \\
(\mathrm{N})\end{array}$ & $\begin{array}{c}\text { Total } \\
(\mathrm{N})\end{array}$ \\
\hline Low & 7 & 31 & 38 & Low & 20 & 45 & 65 \\
\hline High & 9 & 2 & 11 & High & 8 & 3 & 11 \\
\hline \multirow[t]{5}{*}{ Total } & 16 & 33 & 49 & Total & 28 & 48 & 76 \\
\hline & Positive predictive valu & & $18.4 \%$ & \multicolumn{3}{|c|}{ Positive predictive value for low } & $30.8 \%$ \\
\hline & Positive predictive valu & & $81.8 \%$ & \multicolumn{3}{|c|}{ Positive predictive value for high } & $72.7 \%$ \\
\hline & Negative predictive val & & $81.6 \%$ & \multicolumn{3}{|c|}{ Negative predictive value for low } & $69.2 \%$ \\
\hline & Negative predictive valu & & $18.2 \%$ & \multicolumn{3}{|c|}{ Negative predictive value for high } & $27.3 \%$ \\
\hline
\end{tabular}

Table 4. The relationship between the Wells' Criteria s and Revised Geneva Score, and the probability of PTE in pregnant women

\begin{tabular}{lccc}
\hline Wells' Criteria & $\begin{array}{c}\text { CT Positive } \\
(\mathrm{N})\end{array}$ & $\begin{array}{c}\text { CT Negative } \\
(\mathrm{N})\end{array}$ & $\begin{array}{c}\text { Total } \\
(\mathrm{N})\end{array}$ \\
\hline Low & 0 & 13 & 13 \\
Moderate & 4 & 0 & 18 \\
High & 0 & 27 & 0 \\
Total & 4 & 17 & 31 \\
Revised Geneva Score & & 10 & 18 \\
Low & 1 & 0 & 13 \\
Moderate & 3 & 27 & 0 \\
High & 0 & 31 \\
Total & 4 & & \\
\hline
\end{tabular}


PERC rule for patients with possible pulmonary embolism was examined. The results revealed that 2, 32, 45, 28 and 5 patients were scored as 1,2, 3, 4, and 5 respectively.

\section{Discussion}

In general, our results show that $39 \%$ of patients underwent CT angiography diagnosed as pulmonary embolism; this is consistent with other related studies. It does not seem that the usage of this diagnostic modality is more than other centers.

Positive CT angiography test in patients with low, intermediate and high clinical probability was 18,44 , and $82 \%$, respectively, which was better than the results obtained by Vongchaiudomchoke, (7) who reported positive pulmonary CT angiography for high, intermediate and low clinical patients as $59.3 \%, 39.3 \%$ and $7.95 \%$ respectively. Considering the diagnosis of embolism based on clinical and pulmonary angiography test, there is a study called PIOPED II (Prospective Investigation of Pulmonary Embolism Diagnosis II) (8) in which the percentages for high, intermediate and low clinical patients were $92 \%, 96 \%$ and $58 \%$, resopectively. CT angiography was overused in patients with low and intermediate clinical probability. It seems that $\mathrm{CT}$ angiography request needs to be carried out based on clinical decisions made by a doctor taking into account various clinical criteria. It has been suggested that in patients with low clinical probability, the criteria need to be checked and other criteria such as PERC should be used. If PERC is zero, no further action is needed. However, if intermediate and/or low clinical probability plus PERC criteria are above zero, an age adjusted D-Dimer is required, and in case of its positivity, CT angiography is required to be done (9). Stojanovska et al. studied 602 consecutive adult ED patients underwent CTPA for suspected PE and reported that the use of PERC in the ED reduced the use of CTPA significantly and compared with Wells' criteria missed fewer cases of PE, and this is a more efficient tool to make a decision (10).

Among the 31 pregnant patients in our study, 4 were detected to have pulmonary embolism and positive results were not significant compared to those reported by other centers. In another study conducted by Esther et al. (11) on 54 pregnant women undergoing $\mathrm{CT}$ angiography and V/Q scan, from 37 patients underwent CT angiography, 3 were positive, which is almost similar to the results of our study.

Wells' criteria and other similar methods have been known to have low positive predictive value, making their results not valid and reliable for pregnant women (12). Since D-dimer levels are raised in normal pregnancy and early puerperium, present guidelines do not support Ddimer measurement in the evaluation of suspected acute PTE in pregnancy $(13,14)$. Also, there is up to a 10-time increase in VTE incidences in pregnant women compared to non-pregnant women. Side-effects of diagnostic procedures such as breast cancer in mothers who undergo CTPA are more prevalent compared to those who undergo $\mathrm{V} / \mathrm{Q}$, and there is a higher possibility of childhood cancer in V/Q scan compared to CTPA. Therefore, careful decision-making should be made for pregnant women about doing this diagnostic procedure. In the absence of a definitive diagnosis for dyspnea and the presence of clinical suspicion of pulmonary embolism, diagnosis procedure needs to be done with caution- for example bilateral lower limb Doppler ultrasound in pregnant women with suspected pulmonary embolism if they present with signs and symptoms of a DVT or using bismuth breast shield in CTPA which reduces radiation dosage up to $20-40 \%$. If CXR is normal, applying perfusion scan is suggested, which reduces exposure to the fetus $(15,16,17)$.

Our study was a retrospective study based on our hospital records. One of its limitations was lack of follow-up in patients with negative CT pulmonary angiography, and thus we are not sure about the occurrence of pulmonary emboli and DVT after discharge. However, there was no other hospital in the province other than ours to admit patients with pulmonary problems and pulmonary embolism, and we believe in case of vascular problems the patients themselves present to our hospital, or the hospital they initially go, refers them to our hospital which is the only pulmonary center in the province. The second limitation of the study was lack of D-Dimer test for all patients with intermediate and low clinical suspicion. Some of the patients were hospitalized, and CTPA was carried out for them after clinical suspicion. However, we suggest age adjusted D-Dimer Eliza in patients with intermediate and low clinical suspicion if their PERC is positive. None of the studied patients underwent CT pulmonary angiography had zero PERC and thus the patients needed more investigation.

\section{Conclusion}

In conclusion, underuse and overuse of CTPA in diagnosing PTE is a common problem especially in university hospitals. It is possible to avoid unnecessary CTPA requests by more adherence to recommendations of studies and following more accurate clinical risk assessment done by experienced clinicians.

\section{Acknowledgements}

Our special thanks go to all patients who participated in this study.

\section{Conflict of Interests}

The authors declare that they have no competing interests.

\section{References}

1. Gülșen Z, Koşar PN, Gökharman FD. Comparison of multidetector computed tomography findings with clinical and laboratory data in pulmonary thromboembolism. Pol J Radiol. 2015;80:252.

2. Hendriksen JM, Geersing GJ, Lucassen WA, Erkens PM, Stoffers HE, Van Weert HC, et al. Diagnostic prediction models for suspected pulmonary embolism: systematic review and independent external validation in primary care. BMJ. 2015.

3. Kennedy N, Jayathissa S, Healy P. Investigation of suspected pulmonary embolism at Hutt Valley Hospital with CT pulmonary angiography: current practice and opportunities for improvement. Adv Med. 2015;2015.

4. Gu YH, Zhao Z. Role of three commonly used scoring systems in prediction of pulmonary thromboembolism in Xining area. Eur Rev Med Pharmacol Sci. 2014 Nov 1;18(22):3517- 
20.

5. Crichlow A, Cuker A, Mills AM. Overuse of computed tomography pulmonary angiography in the evaluation of patients with suspected pulmonary embolism in the emergency department. Acad Emerg Med. 2012 Nov 1;19(11):1219-26.

6. Molaee S, Ghanaati H, Safavi E, Foroumandi M, Peiman S. Computed tomography pulmonary angiography for evaluation of patients with suspected pulmonary embolism: Use or overuse. Iran J Radiol. 2015 Jul;12(3).

7. Vongchaiudomchoke T, Boonyasirinant T. Positive Pulmonary Computed Tomography angiography in patients with suspected acute pulmonary embolism: clinical prediction rules, thromboembolic risk factors, and implications for appropriate use. J Med Assoc Thai. 2016 Jan 1;99(1):25-33.

8.Stein PD, Fowler SE, Goodman LR, Gottschalk A, Hales CA, Hull RD, et al. Multidetector computed tomography for acute pulmonary embolism. N Engl J Med. 2006 Jun 1;354(22): 2317-27.

9.Raja AS, Greenberg JO, Qaseem A, Denberg TD, Fitterman N, Schuur JD. Evaluation of patients with suspected acute pulmonary embolism: best practice advice from the clinical guidelines committee of the American College of Physicians. Ann Intern Med. 2015 Nov 3;163(9):701-11.

10. Stojanovska J, Carlos RC, Kocher KE, Nagaraju A, Guy K, Kelly AM, et al. CT Pulmonary Angiography . CT pulmonary angiography: using decision rules in the emergency department. J Am Coll Radiol. 2015 Oct 1;12(10):1023-9.

11.Easther S, Langdana F, Beasley R, Maharaj D, Entwisle J, Abels P. The diagnostic role of ventilation/perfusion scans versus computed tomography pulmonary angiography in obstetric patients investigated for pulmonary embolism at Wellington Hospital from 2010 to 2012. N Z Med J. 2016 Apr;129(1433): 62-8.

12. Bourjeily G, Paidas M, Khalil H, Rosene-Montella K, Rodger M. Pulmonary embolism in pregnancy. The Lancet. 2010 Feb 6;375(9713):500-12.

13. Khalafallah AA, Morse M, Al-Barzan AM, Adams M, Dennis A, Bates G, Robertson I, Seaton D, Brain T. D-Dimer levels at different stages of pregnancy in Australian women: a single centre study using two different immunoturbidimetric assays. Thromb Res. 2012 Sep 1;130(3):e171-7.

14. Greer I, Thomson AJ. Green-top Guideline No. 37bthromboembolic disease in pregnancy and the puerperium: acute management. London, BMJ Sex Reprod Health. 2015.

15. Simcox LE, Ormesher L, Tower C, Greer IA. Pulmonary thrombo-embolism in pregnancy: diagnosis and management. Breathe. 2015 Dec;11(4):282.

16. Scarsbrook AF, Evans AL, Owen AR, Gleeson FV. Diagnosis of suspected venous thromboembolic disease in pregnancy. Clin Radiol. 2006 Jan 1;61(1):1-2.

17. Ridge CA, McDermott S, Freyne BJ, Brennan DJ, Collins $\mathrm{CD}$, Skehan SJ. Pulmonary embolism in pregnancy: comparison of pulmonary CT angiography and lung scintigraphy. AJR Am J Roentgenol.2009 Nov;193(5):1223-7. 